\title{
Introduction to Robotics for Novice Users: A Case Study from Summer Schools in Greece
}

\author{
Spyros. E. Polykalas, George N. Prezerakos, Kyriakos G. Vlachos, and Konstantinos Oikonomou
}

\begin{abstract}
The paper conveys experiences and conclusions from the organization of three summer schools aiming at introducing novice users to robotics programming. Issues related to teaching material, teaching approaches, challenges in the classroom and participant's acceptance are all examined in dedicated sections of the paper. The teaching staff's impressions as well as the conclusions drawn from the course evaluation by the participants show that the robots can be successfully employed for introducing novice users, especially schoolchildren, to software development as well as robotic principles.
\end{abstract}

Index Terms - STEM; Robotics; Programing; mBot; Arduino.

\section{INTRODUCTION}

The present paper discusses our experiences from three summer schools organized jointly by the TEI of Ionian Islands, the University of Patras the Piraeus University of Applied Sciences and the Ionian University in 2016 and 2017. The summer schools were held in Argostoli, Cephalonia (2016) and Corfu and Lixouri, Cephalonia (2017) respectively. During the last few years, several efforts are under way in many developed and developing countries with the aim of teaching young children how to code. Good coding skills are considered essential for the economy of the future and countries are trying to prepare early on by introducing related courses in secondary and high school education. On the other hand, teaching software development is especially difficult where young audiences are concerned because most artifacts in software are intangible and thus children cannot easily relate what is conveyed in the classroom with their everyday experience.

The above observation presented a fundamental question that led to the creation of the summer schools project: How one can introduce young children to programming in a direct and understandable manner, obviously without requiring a relevant background in either math or computer science. After spending a significant amount of time researching existing approaches and best practices, it occurred to us that the introduction of a robot as the object of software

Published on February, 2018

S. E. Polykalas is with the Department of Digital Media \& Communication, TEI of Ionian Islands, Greece (e-mail: s.polykalas@teiion.gr).

K. Vlachos is with the Department of Computer Engineering and Informatics, University of Patras, Patras, Greece (e-mail: kvlachos@ceid.upatras.gr).

G. N. Prezerakos is with the Department of Electronic Computer Systems, Piraeus University of Applied Sciences, Piraeus, Greece (e-mail: prezerak@puas.gr).

K. Oikonomou is with the Department of Informatics, Ionian University, Corfu, Greece (e-mail: okon@ionio.gr). development can help us to successfully overcome most of the obstacles related to coding skills teaching. The robot is a real-world object, it can interact with students and with the environment and software behavior becomes immediately obvious in the classroom producing tangible results. In addition, a robot adds a playful dimension in the teaching process which very welcome when one interacts with young audiences. Thus, a decision was taken to organize a series of summer schools focusing on software development for robots and using actual robots the hands-on part of the course.

The paper presents the whole lifecycle of the summer schools project from inception to roll-out. Section 2 provides a more in-depth analysis of the usage of robots on science education. Section 3 discusses the rationale behind choosing a robot for teaching purposes. Section 4 details the structure and philosophy of the teaching material. The subsequent section focuses on the classroom and presents what worked and what didn't with respect to the teaching process. Section 6 contains an analysis of the results of the evaluation that was conducted by the students during the last day of each summer school. Finally, Section 7 summarizes the conclusions drawn from our summer school experience.

\section{RoBOTICS IN STEM EDUCATION}

STEM is a curriculum based program on educating students in four specific disciplines - science, technology, engineering and mathematics - in an interdisciplinary and applied approach. Rather than teaching these four disciplines as discrete subjects, STEM integrates them into a cohesive learning curriculum based on real-world applications, such as robotics. In elementary school, students are still learning with their eyes and hands drawing, molding, and manipulating objects. They are starting the tough transition to learning by reading, and robots make it possible for them to work through problems visually and experiment with concepts they are learning. The adoption of robotics as a mainstream in STEM education was enhanced by the many international competitions, mostly targeting middle and high school students. They position robotics as a "team sport for the mind" and have been successful in developing excitement for robotics and building teamwork skills [1], [2].

The primary objective of educational robotics is to provide the means, usually with an educational robot kit, to facilitate student's development of knowledge, skills and attitudes for the design, analysis and operation in various fields of applications (i.e. industry, home automation, agriculture etc.). In this way, students' interest is excited, keeping highly motivated. 
The term robot here is used quite broadly and may include articulated robots, mobile robots or autonomous vehicles of any scale. The rigor of the approach can be scaled based on the background of the target audience and may be suitable for students across the entire educational spectrum - from elementary school or graduate programs.

Our approach builds on similar efforts aiming at the same goals, carried out in other countries by other researchers [3][5].

\section{Choosing Robots}

Our approach called for a robot that would be easy to program, having attractive (from a usability viewpoint) but not very complicated functions. The robot would ideally carry a price tag that would be within the financial capabilities of the average Greek family in the case where a student, after completing the course, would like to acquire his/her own robot. One additional requirement was that the robot should not be an off the shelf product therefore the student would be required to assemble it into a final product before being able to use it.

This last requirement indicated that our search should concentrate on locating a suitable robotic kit rather than a full-fledged robot. If one searches the Web for the term "robotic kits" he/she will be confronted with more than 40 different solutions. For the summer schools project, we selected the Makeblock DIY mBot Kit [6] since it addressed all our initial requirements. The mBot comes unassembled and consists of a main board, the chassis, wheels and sensors. It can be assembled into a functional robot by a novice user in less than 10 minutes. Programs developed for the mBot are downloaded via Bluetooth or via a wireline USB connection.

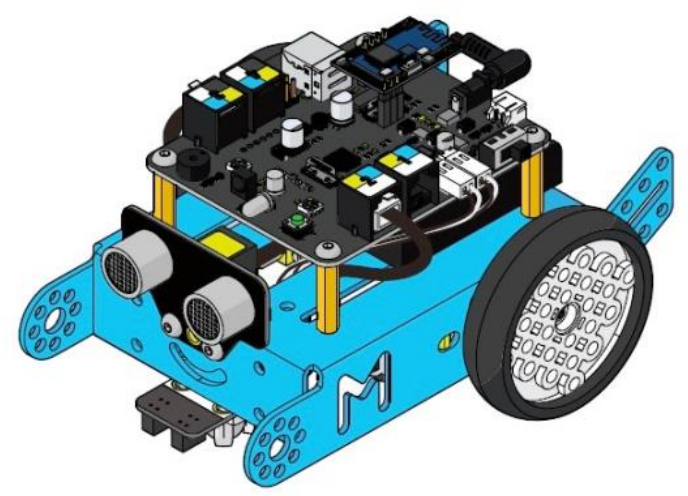

Fig. 1. mBot assembled

Proc Moreover, the mBot had two other very appealing properties. First, it could be programmed with the mBlock programming language which is an extension of Scratch. mBlock provides a visual development environment with an object-oriented approach where one can develop programs for the mBot by customizing and connecting building blocks on the screen. Most children have already some experience with Scratch in their schools. But even those who haven't find it very easy to navigate in the environment and immediately create simple programs by combining the appropriate blocks.

Second, the hardware platform for the mBot is the very popular open source Arduino microcontroller [7]. Thus
Arduino can be used as an introduction to the open source approach of software development as well as for fueling future ideas for development in the field of robotics by the students.

\section{COURSE ORGANIZATION}

Taking all the above into account, ten (10) mBots were procured for use during the summer schools. An additional robot, the Lego EV3RSTORM [8] was added to our collection for demo purposes with the aim to provide the students with a different approach in robot construction as well as a different programming model compared to the mBot. The number of robots was not a random choice but it represented a conscious decision in the sense that with twenty robots we would be able to a support a classroom of up to twenty students, each with his/her own mBot device (in practice, we also had the chance to employ the pair programming model with two students per robot).

The next challenge was to construct a successful curriculum that abided to our initial principle of requiring little or no prior knowledge regarding robotics and programming in general. After a lot of experimentation, it was decided that each school would be conducted during a 4-day period. The first day would include an introduction to robots as well as an additional introduction to basic computer programming concepts (what is a program, variables and control constructs etc.). Following the introduction, the students would have the chance to assemble the robot, get acquainted with the mBlock programming environment and create simple programs that read and display values from the various mBot sensors.

The second day was based on a more complex program that involved mBot movement in the classroom with exercises like avoiding an obstacle, following a line, staying within a bounded area etc. As soon as the students show that they have the ability to tackle these tasks, they are presented with a more complex problem. They are asked to develop a program that will allow the mBot to navigate in a bounded rectangular elongated area containing obstacles, starting from one side and finishing at the other. This task is presented in the form of a contest, students are informed that their robots will participate in a race the next day and the robot that is the first to finish will be the winner. The remainder of the second day as well as a significant part of the third day is spent in developing and testing this program. The mBot part of the summer school ends with the race. The last day of the school is concerned with the Arduino microcontroller and includes a theoretical presentation followed by a series of simple exercises using the board. Again, each student is given his own Arduino board for carrying out the exercises.

\section{EXPERIENCES FROM THE CLASSROOM}

Our impression from the three summer schools organized so far has been fairly positive. Indeed, the usage of mBot is very welcome by the students; it helps in engaging them in the course and is always met with anticipation as well as enthusiasm. The visual programming environment and the mBlock programming language have a very smooth learning curve and in a very short timeframe the students are able to 
tackle the initial exercises. Also, the importance of the mBot race on the third day cannot be stressed enough. Students have the chance to test their code and their robot in a realworld problem, not in isolation, but competing with each other. Everyone is extremely satisfied by taking part in the race regardless of the actual position his/her robot finishes at.

In addition, we must also confess that we were very reluctant regarding the inclusion of a full day devoted to the Arduino microcontroller board taking into account the fact that the majority of students had little or no experience in software development. This was due to the fact that Arduino development involves low level techniques using the $\mathrm{C}$ programming language as well as circuitry and electronics manipulation. Despite our fears, the fourth day has been met with great interest in all three summer schools and there were always a percentage of students that were more keen to work with the board than with the mBot and its programming environment.

On the other hand, there were also difficulties worth noting. The main challenge was that the different students obviously had varying skill sets and learning capabilities. When one student was still struggling with the first programming task of the day, some other could already be one or even two tasks ahead. We addressed this challenge by embracing and, instead of waiting for all students to complete a task before the class could proceed to the next, we allowed each student to work at his/her own pace. In order for this degree of freedom to be supported, at least two tutors should constantly be present in the classroom (assuming a class of 20 students). Finally, an additional interesting observation was that a significant number of students have difficulty in understanding the concept of variables and the concept of logical expressions in branching statements. In every class there are always a small number of students that understand these concepts and put them to use almost immediately. The majority however has to be given several examples employing the robot as well as additional real-world examples before acquiring the ability to incorporate these concepts in their programs.

\section{Evaluation}

On the last day of each summer school, students were asked to participate to an online survey regarding the quality of the course. The issues covered were user related data (age, experience in programming / robotics, level of education) and school related questions (clarity of scope, equipment evaluation, teacher's evaluation, the level of skill improvement attained and willingness to attend another summer school in the future). In the following figures aggregate the students' answers from all three summer schools and a qualitative analysis is performed.

Looking at the age of participants (Fig. 2), we observe that almost half of the students are under the age of 18 . Another significant group is those belonging to ages between 19 - 35. Finally, a small percentage (slightly over $10 \%$ ) corresponded to 36 - 55 years of age.

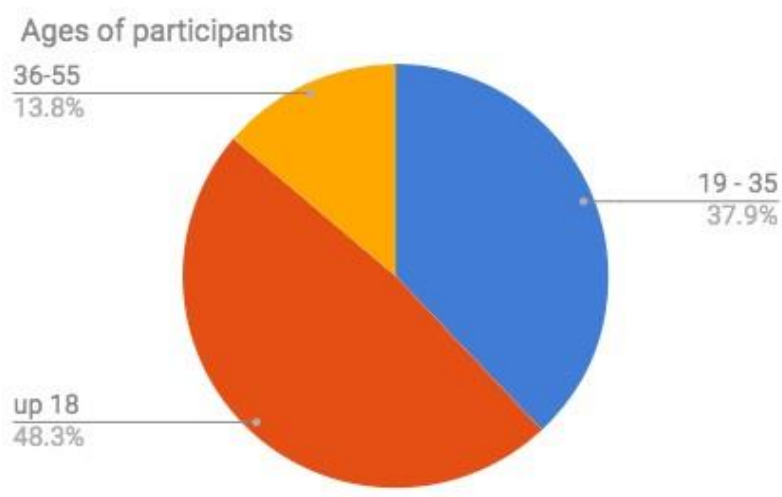

Fig. 2. Age of participants

With respect to experience in programming (Fig. 3), the three main groups are comprised of students with HTML knowledge, students acquainted with Scratch and students with some experience in HTML, C and Java. Other programming languages are also represented but to a lesser extent. Scratch and HTML are present obviously because they are taught to some extent in Greek primary and secondary education.

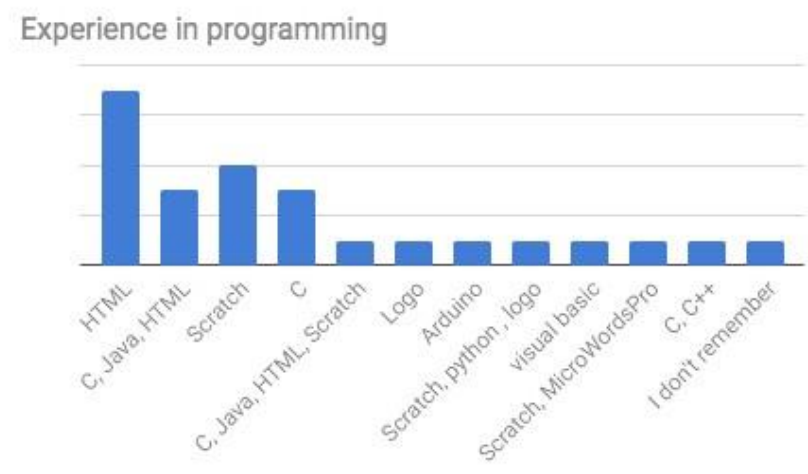

Fig. 3. Experience in programming

The majority of the participants are elementary school students, with University students constituting the second most significant batch (Fig. 4).

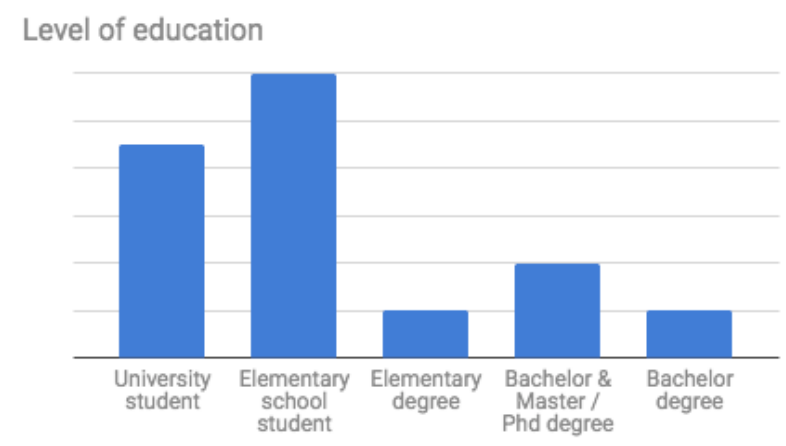

Fig. 4. Level of education

Finally, an impressive $72 \%$ of the participants did not have any prior experience in robotics. (Fig. 5) 


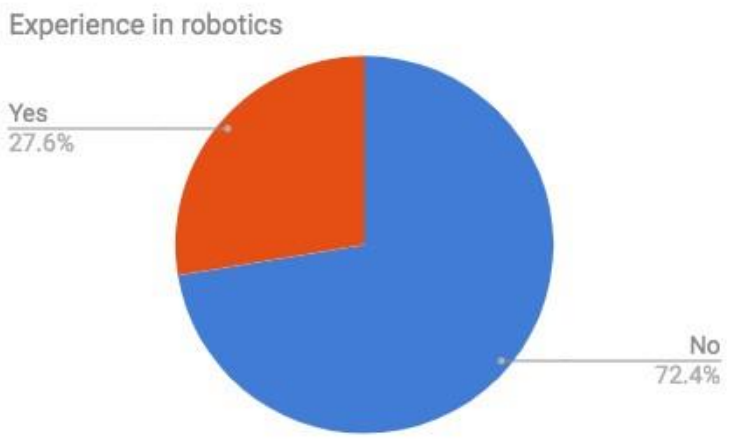

Fig. 5. Experience in robotics

In regard to the evaluation of the course itself (Fig. 6), all three main criteria (clarity of scope, equipment, and teachers) were highly evaluated. In addition, most students felt that the summer school helped them to improve their computer programming skills.

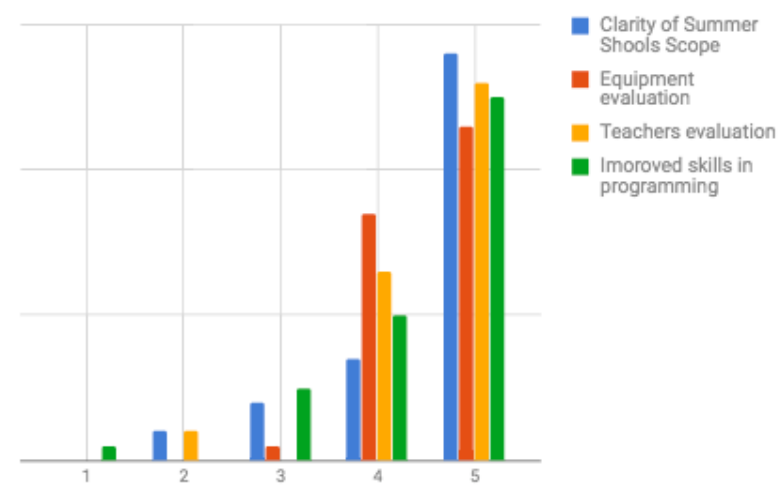

Fig. 6. Course evaluation

All four main legs of the course (Theory, mBot, Arduino and Lego) were well received (Fig. 7). However, Lego lags behind the other three and was the only session that had some students rating it as "Poor". To our defense, we must restate the Lego was more of a demo session that a regular training session in robotics. Since it does not seem to rate well, there are thoughts to exclude it entirely from future summer schools or to organize a 4-day summer school including only Lego programming.

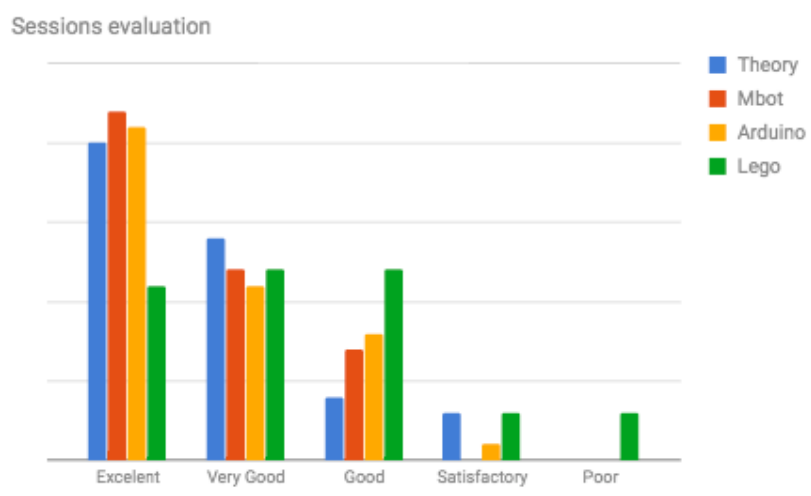

Fig. 7. Sessions evaluation

Finally, an astounding $80 \%$ of the participants expressed their willingness to participate in another robotics summer school in the future.

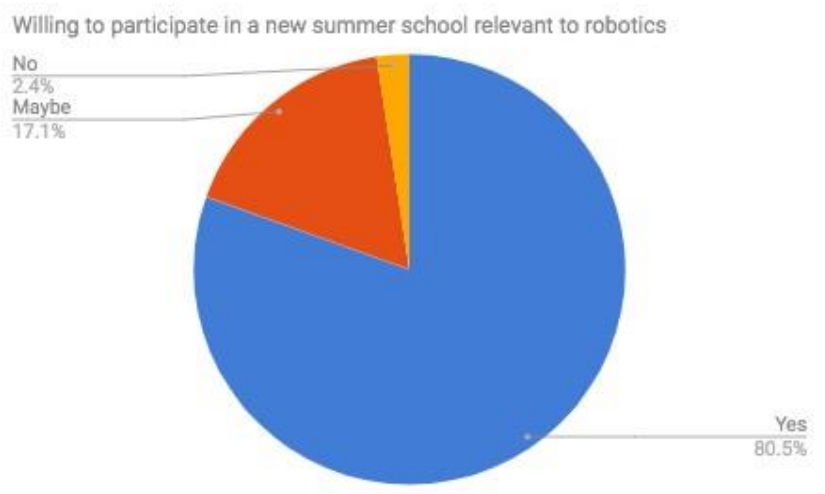

Fig. 8. Willingness to participate again

\section{CONCLUSIONS}

Our journey so far in the world of robotics summer schools has convinced us that robotic kits are an ideal vehicle for introducing novice users, especially schoolchildren, to computer programming. Successful planning represents the most important success factor especially in the areas of selecting a robotic kit, constructing an appropriate curriculum and employing a teaching approach that allows each student to work at a pace that is most convenient for him/her. In our case, this effort paid off, judging from the evaluation results. From the teaching staff's viewpoint, it was a very positive experience as well and we are looking forward to many more robotic summer schools in the future.

\section{REFERENCES}

[1] A. Eguchi, "RoboCupJunior for promoting STEM education, 21st century skills, and technological advancement through robotics competition," Rob. Auton. Syst., vol. 75, pp. 692-699, 2016.

[2] S. Nag, J. Katz, and A. S.-O. Acta, "Collaborative gaming and competition for CS-STEM education using SPHERES Zero Robotics," Acta Astronaut., vol. 83, pp. 145-174, 2013.

[3] M. Mataric, N. Koenig, and D. Feil-Seifer, "Materials for Enabling Hands-On Robotics and STEM Education.," in AAAI spring symposium: Semantic scientific knowledge integration, 2007, pp. 99102.

[4] G. Nugent, B. Barker, N. Grandgenett, and V. I. Adamchuk, "Impact of Robotics and Geospatial Technology Interventions on Youth STEM Learning and Attitudes," J. Res. Technol. Educ., vol. 42, no. 4, pp. 391-408, Jun. 2010.

[5] M. Bers, S. Seddighin, and A. S.-J. Teacher, "Ready for robotics: Bringing together the T and E of STEM in early childhood teacher education," J. Technol. Teach. Educ. 21(3), 355-377., vol. 21, no. 3, pp. 355-377, 2013.

[6] mBot robotic kit, "http://learn.makeblock.com/en/mbot/," 2017.

[7] A. Microcontroller, "https://www.arduino.cc/," 2017.

[8] Lego Ev3rstorm, "https://www.lego.com/en-us/mindstorms/build-arobot/ev3rstorm," 2017.

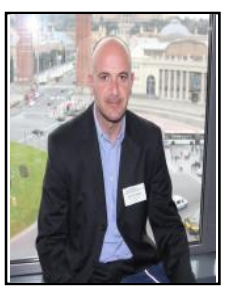

Spyros E. Polykalas received his diploma degree (Dipl.-Ing.) in electrical and computer engineering from the University of Patras in 1994, and Doctoral Degree in 1999 from National Technical University of Athens (NTUA).

From 1999 until 2001 he was a research associate in NTUA and from 2001 to 2008 he worked as Special Scientist in Greek Telecommunication Regulatory Authority (EETT). In 2008 joined the faculty of the Department of Business Administration of the Technological Educational Institute of Ionian Islands, as an Assistant Professor. From 2014 holds a position of an Associate Professor in the Department of Digital Media and Communication. His main scientific interests are related to the provision of Electronic Communication Services. In particular he has more than 20 years of experience in issues such as 
performance evaluation of Intelligent Broadband Networks, economictechnical models of telecommunications networks, legal/regulatory framework for telecommunications and information technology, regulatory policy of EU on the development of competition in electronic communications and personal data privacy.

Prof. Polykalas has been participated in several national and international projects related to provision of electronic communication services. He is a co-author of two scientific books, while his research work in the above mentioned fields has been published in international journals and has been presented in international conferences.

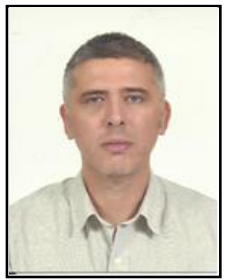

Dr. George N. Prezerakos was born in Athens, Greece, in December 1970. He earned an M.Sc. from the National Technical University of Athens (NTUA - Electrical and Computer Engineering Dept), in 1993 and a Ph.D in 1998 also from NTUA in the area of Software Engineering for Broadband Services. From 1993 to 1998 he was a research associate of the NTUA Telecommunications Laboratory performing research on Intelligent Broadband Networks, Service Engineering and Fuzzy Logic Systems.

Consequently, he has held various positions in the Greek software and telecoms market and in the Greek Telecommunication Regulatory Authority (EETT). He is currently a Professor in the Dept. of Computer Systems Engineering, Piraeus University of Applied Sciences, Greece. His current research interests are in the fields of service and platform engineering, context-aware services, model driven development and telecoms regulation. He has several publications in the above areas.

Dr. George N. Prezerakos has participated in several European Union and national projects. He is member of the Technical Chamber of Greece.

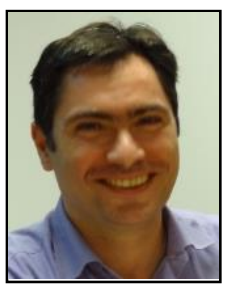

Kyriakos G. Vlachos, received his Dipl.-Ing. degree in electrical and computer engineering from the National University of Athens (NTUA), Greece, in 1998 and his Ph.D. in electrical and computer engineering, also from NTUA, in 2001.

From 1997 to 2001 he was a Senior Research Associate in the Photonics Communications Research Laboratory (ICCS/NTUA). In 2001, he joined Bell Laboratories, Lucent Technologies, working in the Applied Photonics Group. Since 2003, he had been also a member of the Computer Engineering Laboratory of the Technical
University of Delft, and since 2005 he has been a Faculty Member of the Computer Engineering and Informatics Department of the University of Patras, Greece. His research interests are in the areas of architectures and technologies for broadband, high-speed networks.

Prof. Vlachos has participated in various research projects funded by the European Commission (IST-STOLAS, IST-PRO3, ESPRIT-DOALL, ephoton/ONe+, ICT-BONE, and ICT-DICONET). He is a member of IEEE and the Technical Chamber of Greece. He is the (co) author of more than 120 journal and conference publications and also holds two international patents.

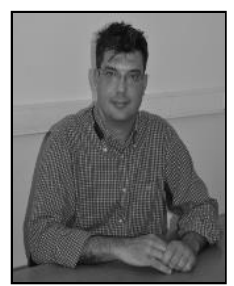

Konstantinos Oikonomou has received his diploma in Computer Engineering and Informatics from University of Patras in 1998. In September 1999 he received his postgraduate degree: M.Sc. in Communication and Signal Processing from the Electrical and Electronic Engineering Department, Imperial College (London). He received his Ph.D. degree in 2004 from the Informatics and Telecommunications Department, University of Athens, Greece. His Ph.D. thesis focuses on medium access control policies in ad hoc networks.

$\mathrm{He}$ is currently the Dean of the Faculty of Information Science and Informatics of the Ionian University, Corfu, Greece. Since 2006 he is a faculty member in Computer Networks at the Department of Informatics ofn the same university. He is currently an Associate Professor and has also served as the Head of the Department. Between December 1999 and January 2005 he was employed at Intracom S.A, as a research and development engineer and in 2005. His current interests involve medium access control in ad hoc networks, performance issues in wireless networks, information dissemination, service discovery, facility location, energy consumption in cloud computing environments.

Dr. Oikonomou has a long experience regarding wireless systems and he has been co-ordinating a number of EC research projects in the computer networks research area and in various local development projects (e.g., virtual worlds). He has been a reviewer and TPC member of numerous conferences and journals in the area. He holds an award for the best paper from the Hawaii International Conference on System Service and another one from the Hellenic Mathematical Society 